\title{
Skin Reactions to Thimerosal and Leishmania in Dogs from a Leishmaniasis Endemic Area: it is Better to Keep them Apart
}

\author{
Moacir Paranhos-Silva, Lain C Pontes-de-Carvalho/*, Geraldo G de Sá \\ Oliveira, Eliane Góes Nascimento**, Washington LC dos-Santos/*/+
}

\begin{abstract}
Centro de Pesquisas Gonçalo Moniz-Fiocruz, Rua Valdemar Falcão 121, 40295-001 Salvador, BA, Brasil
*Escola Bahiana de Medicina e Saúde Pública, Salvador, BA, Brasil **Centro de Referência em Doenças Endêmicas Pirajá da Silva, Jequié, BA, Brasil
\end{abstract}

\begin{abstract}
Positive Montenegro's skin test is a delayed type hypersensitivity reaction widely used as indicative of previous infection with Leishmania in both humans and dogs. Montenegro's antigen consists of a crude Leishmania antigen solution, usually containing thimerosal as preserving agent. In this work it is shown that a large proportion of dogs (11 out of 56) examined in an endemic area of leishmaniasis presented induration at the site of injection of a diluent containing thimerosal alone. This clearly demonstrates that thimerosal leads to a high number of false positive skin reactions in dogs and that its use in Montenegro's skin test antigenic preparations should be avoided.
\end{abstract}

Key words: Montenegro's skin test - Leishmania - delayed type hypersensitivity - thimerosal -

canine visceral leishmaniasis

The Montenegro's skin test (MST) has been used as indicative of previous infection with Leishmania (Melo et al. 1977) and of a cellular immune reaction against the parasite (Mayrink et al. 1989, Barral et al. 1997). Although the test was originally designed for humans, published data indicate that it may be successfully adapted for dogs (Marzochi \& Barbosa-Santos 1988, Genaro et al. 1992, Hermeto et al. 1993) and it has been used as a marker of protective immunity in recent studies (Pinelli et al. 1994). The antigens normally used in these tests are crude promastigote antigens produced by sonication of parasites, and preserved with thimerosal or phenol (Neal \& Miles 1976, Melo et al. 1977, Pinelli et al. 1994).

In a recent survey of canine population in Jequié, State of Bahia, an area endemic for visceral leishmaniasis, we tested 17 dogs for their immune reactivity to Leishmania, through ELISA

This work was supported by the Brazilian National Research Council, CNPq, grant no. 521260/98-1, and Pronex.

${ }^{+}$Corresponding author. Fax: + 55-71-356.2255. E-mail: wluis@svn.com.br

Received 30 August 2000

Accepted 13 December 2000 and MST, using antigens from different Leishmania species. Antigenic homogenates were prepared from L. major (MHOM/IR-173), L. amazonensis (Leila strain, MHOM/BR/88/BA-125), L. braziliensis (MOM3456) and L. chagasi (Merivaldo strain, IOC-LC2455, isolated from a patient with visceral leishmaniasis in the endemic area of Jequié) stationary-phase promastigotes, by ultrasound treatment at $4^{\circ} \mathrm{C}$. A L. amazonensis (BH8 strain) homogenate, widely used by the Brazilian National Health Service, was produced by Bio-Manguinhos (Oswaldo Cruz Foundation, Rio de Janeiro, Brazil). All antigens were diluted in saline, to a final concentration of $2 \mathrm{mg}$ of protein/ ml. Thimerosal (Sigma Chemical Co., St. Louis, USA; $1 / 10,000$ dilution), used as a preserving agent in the Bio-Manguinhos antigen, was also added to some of the antigens produced in our laboratory. Leishmania homogenates without thimerosal did not produce skin indurations in any of the 17 tested dogs from a Leishmania-free area (Salvador-Brazil). Dogs were collected in the streets of Jequié by the canine surveillance program of the Municipal Health Service. All animals $(n=17)$ were tested for presence in serum (diluted 1:100) of antibodies against Leishmania by ELISA (Silva et al. 1996). In the following day each animal was injected with $100 \mu \mathrm{l}$ volumes of each antigen solution or the diluting solution (saline containing $1 / 10,000$ thimerosal), at different sites in the ab- 
dominal skin. Forty-eight hours after injection the size of induration was measured by one of us, without previous knowledge of the sites of injection of antigens or diluent. Skin reactions with diameter equal or larger than $5 \mathrm{~mm}$ were considered positive. The animals were killed in the following day and spleen samples were placed in biphasic medium for the possible growth of Leishmania. Six animals (35\%) had induration at the sites of injection of Leishmania antigen (Table). However, in three of these six animals (17\% of the animals) there was also an induration with $5 \mathrm{~mm}$ diameter or larger at the site where the thimerosal-containing diluent was injected. One of the dogs responded only to $L$. braziliensis antigen (L. braziliensis infection is endemic in rural areas of Jequié). One out of 6 animals with induration, and 4 out of 11 animals without induration, had positive culture for Leishmania.
In a subsequent study in the same endemic area, antigenic preparations without thimerosal were also used. In this study the response to Leishmania antigens or thimerosal could be better discriminated: 7 out of 39 animals (18\%) had positive MST and no induration at the site of thimerosal free-diluent injection (Table). Two among these 7 animals with positive MST, and 6 with negative MST, developed induration with $5 \mathrm{~mm}$ or larger at the site of thimerosal injection (20\% of the animals in this second experiment). In 3 animals in which the histological aspects of the lesions induced by thimerosal injection were examined, an inflammatory infiltrate constituted predominantly by macrophages with large granular cytoplasm, sometimes exhibiting an aspect of foam cells was observed. Some lymphocytes and few neutrophils, associated with necrotic cells, were present. Six out of the 7

TABLE

Montenegro's skin test (MST), using different antigens, in mongrel dogs from Jequié, State of Bahia, Brazil

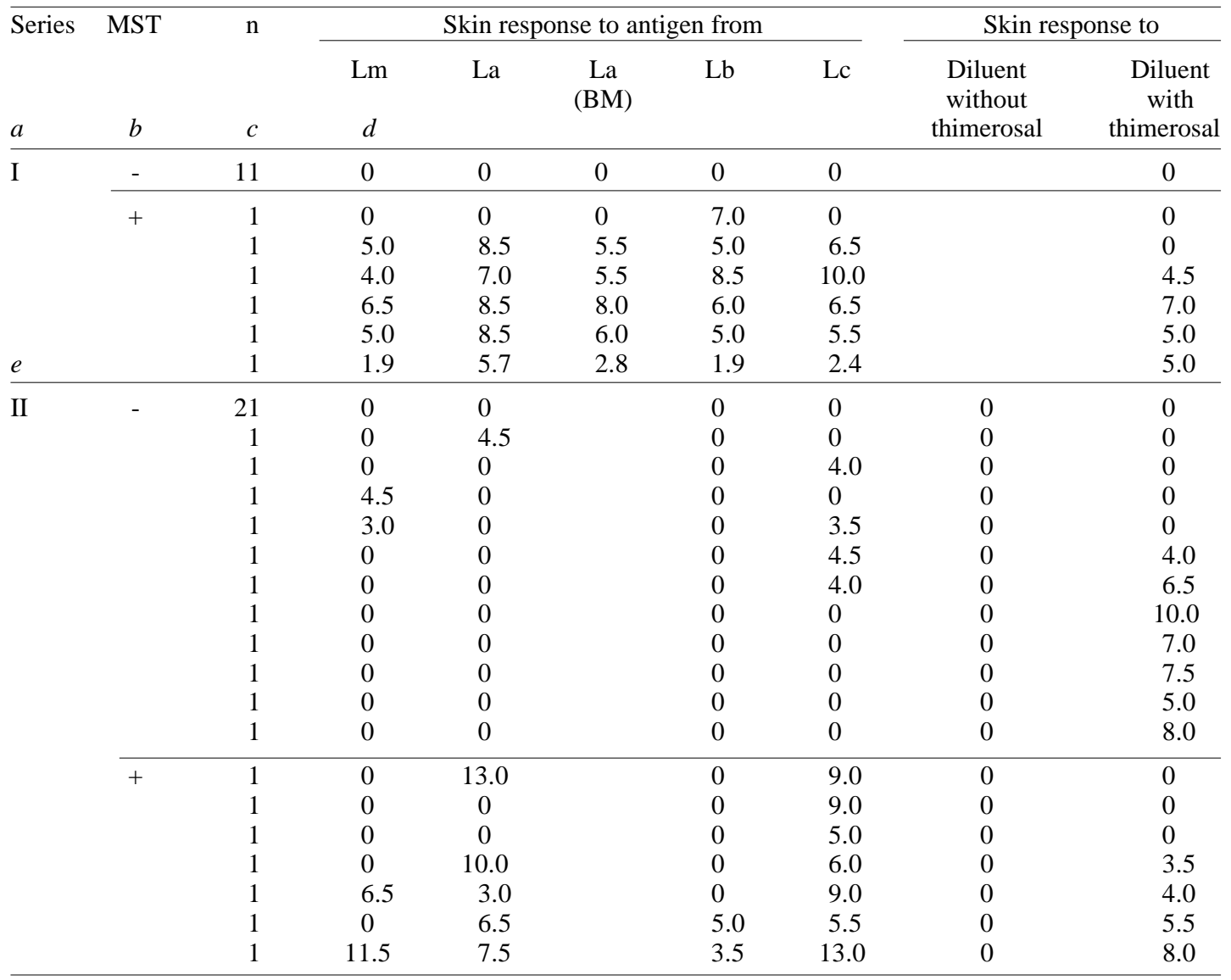

Lm: Leishmania major; La: L. amazonensis; La (BM): L. amazonensis from Bio-Manguinhos; Lb: L. braziliensis; Lc: L. chagasi; $a$ : all antigens used in this series contained thimerosal; $b$ : diameter of induration smaller than $5 \mathrm{~mm}$ were considered as being negative; $c$ : number of dogs with a particular quantitative/qualitative pattern of skin reactivity to antigens and diluent; $d$ : diameter of induration area in $\mathrm{mm} . e$ : antigens used in this series did not contain thimerosal. 
animals with positive MST test had positive ELISA and only 1 out of 7 had positive spleen cultures. In the group without positive MST, 25 out of 32 had positive ELISA and 6 out of 32 animals had positive culture for Leishmania. Although the proportion of dogs with positive spleen culture in the group with positive MST was low (1 out of 7), it did not differ statistically from that observed in the group with negative MST but with other evidence of Leishmania infection such as positive serology and/or presence of Leishmania in spleen cultures ( 6 out of $25 ; p=0.511$, Fisher's exact probability test). Although not statistically significant, such a relatively low proportion of animals with positive spleen cultures in the group with positive MST is in agreement with the observation by other authors (Pinelli et al. 1994) that positive delayed type hypersensitivity against Leishmania antigens may reflect, also in dogs, a protective state against the infection. The presence of positive spleen culture in one of the animals with positive MST in our series, however, suggests that positive delayed type hypersensitivity may not be used as a single parameter of protection in canine visceral leishmaniasis, and that other immunological and clinical parameters must be associated to MST in order to ascertain the status of susceptibility/resistance of dogs to Leishmania infection.

The proportion of dogs reacting to thimerosalcontaining diluent (11 out of 56 in the two series) was significantly higher ( $\mathrm{p}=0.002$, Fisher's exact probability test) than the proportion of those reacting to diluent without thimerosal (0 out of 39). This work draws attention to the fact that thimerosal, widely used in preparation of Montenegro's antigen, leads to a high number of false positive results in dogs and that its use in MST antigenic preparations should be avoided.

\section{REFERENCES}

Barral-Netto M, Machado P, Bittencourt A, Barral A 1997. Recent advances in the pathophysiology and treatment of human cutaneous leishmaniasis. Current Opinion Dermatol 4: 51-58.

Genaro O, Raso P, Costa CA, Carvalho MD, Amaral F, Botelho ACC, Williams P, Dias M, Mayrink W 1992. Montenegro skin tests in dogs experimentally infected with Leishmania (Viannia) braziliensis. Mem Inst Oswaldo Cruz 87: 163-164.

Hermeto MV, Vieira-Dias D, Genaro O, Costa CA, Toledo VPCP, Michalick MS, Tafuri WL, Chaves KM, Mayrink W 1993. Delayed hypersensitivity skin-test using leishvacin for epidemiological survey of canine cutaneous leishmaniasis in a rural area of Minas Gerais. Mem Inst Oswaldo Cruz 88: 635-536.

Marzochi MCA, Barbosa-Santos EGO 1988. Evaluation of a skin test on the canine munocutaneous leishmaniasis diagnosis. Mem Inst Oswaldo Cruz 83: 391392.

Mayrink W, Schettini AP, Williams P, Raso P, Magalhães PA, Lima AO, Melo MN, Costa CA, Genaro O, Dias M, Michalick MSM 1989. Histological observations on Montenegro's reaction in man. Rev Inst Med Trop S Paulo 31: 256-261.

Melo MN, Mayrink W, da Costa CA, Magalhães PA, Dias M, Williams P, Araújo FG, Coelho MV, Batista SM 1977. Padronização do antígeno de Montenegro. Rev Inst Med Trop São Paulo 19: 161-164.

Neal RA, Miles RA 1976. The Montenegro reaction in guinea pigs infected by Leishmania enriettii and the effect of antigens prepared from various Leishmania isolates. Am J Trop Med Hyg 79: 32-37.

Pinelli E, Killick-Kendrick R, Wagenaar J, Bernadina W, del Real G, Ruitenberg J 1994. Cellular and humoral immune responses in dogs experimentally and naturally infected with Leishmania infantum. Infect Immun 62: 229-235.

Silva MP, Freitas LAR, Santos WLC, Grimaldi-Jr G, Pontes-de-Carvalho LC, Oliveira-dos-Santos AJ 1996. A cross-sectional serodiagnostic survey of canine leishmaniasis due to Leishmania chagasi.Am J Trop Med Hyg 55: 39-44. 